\title{
Waning population immunity prior to a large $Q$ fever epidemic in the south of The Netherlands
}

\author{
D. A. H. BRANDWAGT ${ }^{1,2 *}$, T. HERREMANS ${ }^{1}$, P. M. SCHNEEBERGER ${ }^{3}$, \\ V. H. HACKERT ${ }^{4,5}$, C. J. P. A. HOEBE ${ }^{4,5}$, J. PAGET ${ }^{6}$ AND W. VAN DER HOEK ${ }^{1}$ \\ ${ }^{1}$ National Institute for Public Health and the Environment (RIVM), Centre for Infectious Disease Control, \\ Bilthoven, The Netherlands \\ ${ }_{2}^{2}$ Municipal Health Service (GGD) region Utrecht, Zeist, The Netherlands \\ ${ }^{3}$ Department of Medical Microbiology and Infection Control, Jeroen Bosch Hospital, 's-Hertogenbosch, \\ The Netherlands \\ ${ }_{5}^{4}$ South Limburg Public Health Service, Geleen, The Netherlands \\ ${ }^{5}$ Maastricht University Medical Centre, School of Public Health and Primary Care, Maastricht, The Netherlands \\ ${ }^{6}$ Netherlands Institute for Health Services Research (NIVEL), Utrecht, The Netherlands
}

Received 23 July 2015; Final revision 11 February 2016; Accepted 22 March 2016;

first published online 14 April 2016

\section{SUMMARY}

Historical survey data suggest that the seroprevalence of antibodies against Coxiella burnetii in the general population of The Netherlands decreased from more than $40 \%$ in 1983 to $2.4 \%$ in 2007, just before the start of the large 2007-2010 Q fever epidemic. To assess whether the sharp decline in seroprevalence was real, we performed a cross-sectional study using historical samples. We tested samples using a contemporary commercial indirect immunofluorescence assay. In plasma samples from the south of The Netherlands from 1987, we found an age- and sexstandardized seroprevalence of $14 \cdot 4 \%(95 \%$ confidence interval $11 \cdot 2-18 \cdot 3)$. This was significantly lower than a 1983 estimate from the same area $(62 \cdot 5 \%)$, but significantly higher than $2008(1 \cdot 0 \%)$ and $2010(2 \cdot 3 \%)$ estimates from the same area. The study suggests that there was a steady and sharp decline in Q fever seroprevalence in the south of The Netherlands from 1987 to 2008. We assume that seroprevalence has decreased in other parts of The Netherlands as well and seroprevalence surveys in other European countries have shown a similar declining trend. Waning population immunity in The Netherlands may have contributed to the scale of the 2007-2010 Q fever epidemic. For a better understanding of the infection dynamics of Q fever, we advocate an international comparative study of the seroprevalence of $C$. burnetii.

Key words: Coxiella, epidemiology, infectious disease epidemiology, Q fever, zoonoses.

\section{INTRODUCTION}

Q fever is a globally occurring zoonosis caused by Coxiella burnetii, an intracellular Gram-negative bacterium. The main reservoirs for $\mathrm{Q}$ fever in humans are

\footnotetext{
* Author for correspondence: D. A. H. Brandwagt, MD, GGD Region, Utrecht, PO Box 51, 3700 AB Zeist, The Netherlands. (Email: dbrandwagt@ggdru.nl)
}

domestic ruminants, such as cattle, goats and sheep. Transmission to humans mainly occurs through inhalation of contaminated aerosols, released when infected animals give birth [1].

From 2007 to 2010, The Netherlands faced increasingly large annual and seasonal outbreaks of $\mathrm{Q}$ fever. More than 4000 acute human Q fever cases were notified, making this the largest recorded $\mathrm{Q}$ fever epidemic worldwide to date [2]. Although no outbreaks 
of Q fever had been reported in The Netherlands prior to 2007 , historical data suggest high endemicity in preceding decades. In sera of blood donors from various regions of The Netherlands in 1968 and 1983, high seroprevalences were found [3], significantly higher than the $2 \cdot 4 \%$ seroprevalence in the general population of The Netherlands in 2006-2007, just prior to the first Q fever outbreak [4].

However, comparison of findings from different surveys may be problematic, due to discrepancies in the populations surveyed and the laboratory methods used. For humans, population-based seroprevalence studies routinely measure IgG phase II antibodies. Indirect immunofluorescence assay (IFA) is regarded as the reference test for serological detection of Q fever. However, performing IFA is labour intensive and there is no uniform consensus for the cut-off titres. For large studies, ELISA is a convenient test as it can be easily automated. However, ELISA may be less sensitive than IFA [5].

We analysed the seroprevalence dynamics of $\mathrm{Q}$ fever before and during the recent Dutch epidemic. We used historical and recently acquired seroprevalence data, taking into account the dilemma of using different diagnostic tests and cut-off definitions.

\section{MATERIALS AND METHODS}

\section{Study design and study area}

We performed a cross-sectional study using historical samples from 1987, analysed with a commercial IFA. The results were compared with historical results from 1983 to assess the accuracy of the historical data. Furthermore, the results were compared with recently acquired data from 2008 and 2010. All samples and data were collected from the same geographical area, i.e. South Limburg, the most southern part of the province of Limburg, The Netherlands (Fig. 1).

This region had 606000 inhabitants in 2014 [data from Statistics Netherlands (CBS)] combining both urban and rural areas. With 917 population $/ \mathrm{km}^{2}$, it is one of the most densely populated regions of The Netherlands. The largest cities in the region are Maastricht, Heerlen and Sittard. In 2009, the South Limburg region faced a large outbreak of $\mathrm{Q}$ fever around the municipality of Voerendaal originating from a dairy goat farm [6].

\section{Study population}

The samples for 1987 were available from the biobank of the Monitoring Project on Cardiovascular Risk

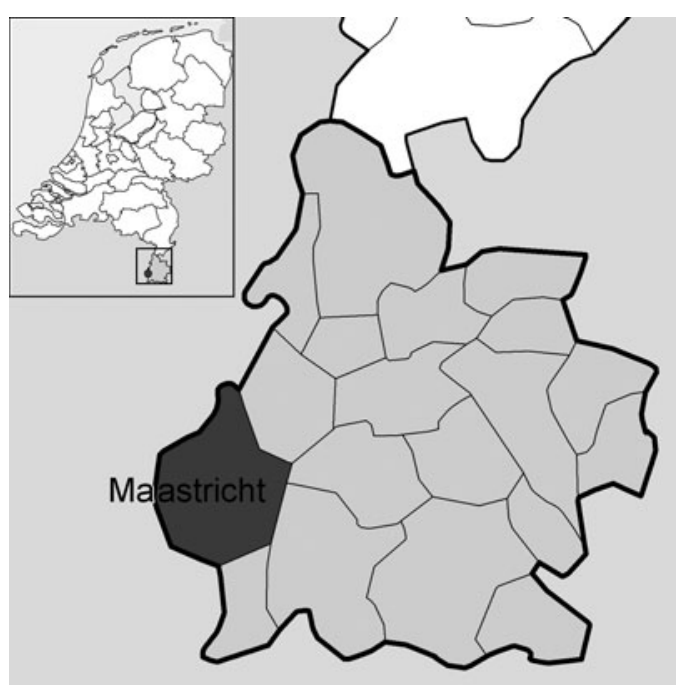

Fig. 1. Map of South Limberg region with the municipality of Maastricht highlighted.

Factors. This project was conducted in The Netherlands from 1987 to 1991 . More than 36000 persons aged 20-59 years were screened for cardiovascular risk factors, using questionnaires and blood samples [7]. The survey was conducted in the municipalities of Amsterdam, Doetinchem and Maastricht. Stored plasma samples from this survey were available for further research and had not been tested previously for $\mathrm{Q}$ fever or other infectious diseases. A random selection of plasma samples from 1987 was taken from the Maastricht subgroup. Assuming an estimated seroprevalence of $50 \%$ with a confidence level of $95 \%, 385$ samples would be required for adequate power. The samples had been stored at $-35^{\circ} \mathrm{C}$ in the biobank at the centres for Nutrition and Health (CVG) and Prevention and Health Services Research (PZO) at the National Institute for Public Health and the Environment (RIVM). The use of the anonymous plasma samples was approved by the Scientific Advisory Group of the PZO.

For the 1987 samples, antibodies against C. burnetii were determined in plasma by IFA (Focus Diagnostics, USA) measuring IgG against $C$. burnetii phases I and II according to the manufacturer's instructions. Seropositivity was defined as an IgG phase II titre $\geqslant 1: 32$. The plasma samples were analysed at RIVM.

\section{Comparison populations}

Data from 1983 were available from the Maastricht subgroup in a seroprevalence survey performed by Richardus et al. [3]. In this survey, plasma samples 
Table 1. Prevalence of IgG antibodies against phase II of Coxiella burnetii in samples from Maastricht and South Limburg

\begin{tabular}{|c|c|c|c|c|c|c|c|}
\hline \multirow[b]{2}{*}{ Year } & \multicolumn{2}{|c|}{ Male } & \multicolumn{2}{|c|}{ Female } & \multicolumn{2}{|c|}{ Total } & \multirow{2}{*}{$\begin{array}{l}\text { Adjusted seroprevalence* } \\
(95 \% \text { CI })\end{array}$} \\
\hline & $N$ & Pos, $n(\%)$ & $N$ & Pos, $n(\%)$ & $N$ & Pos, $n(\%)$ & \\
\hline 1983 & 125 & $74(59 \cdot 2)$ & 123 & $79(64 \cdot 2)$ & 248 & $153(61 \cdot 7)$ & $62 \cdot 5(56 \cdot 3-68 \cdot 3)$ \\
\hline 1987 & 176 & $26(14 \cdot 7)$ & 204 & $31(15 \cdot 2)$ & 380 & $57(15 \cdot 0)$ & $14 \cdot 4(11 \cdot 2-18 \cdot 3)$ \\
\hline 2008 & 181 & $2(1 \cdot 1)$ & 666 & $6(0 \cdot 8)$ & 847 & $7(0 \cdot 8)$ & $1 \cdot 0(0 \cdot 5-1 \cdot 9)$ \\
\hline 2010 & 290 & $6(2 \cdot 1)$ & 343 & $11(3 \cdot 2)$ & 633 & $17(2 \cdot 7)$ & $2 \cdot 3(1 \cdot 4-3 \cdot 8)$ \\
\hline
\end{tabular}

CI, Confidence interval.

* Seroprevalence standardized for age distribution and gender, based on population data from Statistics Netherlands (CBS).

from 248 not randomly selected blood donors were screened using an in-house developed IFA. Seroprevalence data for 2008 and 2010 were available from a survey that was conducted in 2010 in response to the large outbreak of Q fever in South Limburg [6]. In this study, 847 samples from 2008 of adults vaccinated for healthcare-related hepatitis B risk and 633 samples from 2010 of persons attending the regional sexual health clinic from January to April 2010 were screened for antibodies against $C$. burnetii, using a commercial ELISA (Institute Virion/Serion GmbH, Germany). Positive and intermediate results were confirmed using an IFA (Fuller Laboratories, USA) at a cut-off value of 1:16, according to the manufacturer's instructions.

\section{Statistical analysis}

The samples for 1987, 2008 and 2010, were divided into the same five age groups that were used in the 1983 survey of Richardus et al. [3]. For each year, the results were directly standardized for age group and gender, using demographic data for The Netherlands for the respective year as published by Statistics Netherlands. For the years 1983, 1987 and 2008 (the years without recent outbreaks), the seroprevalence figures by age were also visualized using error bar diagrams, representing the confidence interval (CI) of the mean seroprevalences. For data analysis SPSS Statistics v. 21 (IBM Corp., USA) was used.

\section{RESULTS}

Of the 385 selected plasma samples from 1987, 380 were suitable for analysis. The samples were from $176(46 \%)$ male and 204 (54\%) female participants. Of the 380 samples, 57 tested positive for antibodies against $C$. burnetii. When standardized for age and gender, this resulted in a seroprevalence of $14 \cdot 4 \%(95 \%$ CI $11 \cdot 2-18 \cdot 3)$.
The 1983 study included $125(50 \%)$ male and 123 $(50 \%)$ female participants [3]. Of the 248 samples, 153 tested positive for antibodies against C. burnetii, resulting in an age- and sex-standardized seroprevalence of $62 \cdot 5 \%(95 \%$ CI $56 \cdot 3-68 \cdot 3)$.

The 2008 samples included $181(21 \%)$ male and 666 (79\%) female participants. Of the 847 samples, seven samples tested positive for antibodies against $C$. burnetii, resulting in an age- and sex-standardized seroprevalence of $1 \cdot 0 \%(95 \%$ CI $0 \cdot 5-1 \cdot 9)$.

The 2010 samples included 290 male $(46 \%)$ and 343 (54\%) female participants. Seventeen of the 633 samples tested positive for antibodies against $C$. burnetii, resulting in an age- and sex- standardized seroprevalence of $2 \cdot 3 \%(95 \%$ CI $1 \cdot 4-3 \cdot 8)$.

Table 1 shows the crude and standardized seroprevalence results for all four populations. None of the surveys had statistically significant differences in seroprevalence between age groups or between males and females. A graphical comparison of the seroprevalence estimates from 1983, 1987 and 2008 is shown in Figure 2. The seroprevalence estimates for 1987 are significantly lower than the seroprevalence estimates from 1983 across all age groups. The seroprevalence in 1987 was significantly higher than the seroprevalence in 2008 for all age groups, except the 20-24 years age group (Fig. 2). The difference in seroprevalence between 2010 and 2008 was not statistically significant.

\section{DISCUSSION}

The present study, using stored samples from 1987, confirms that seroprevalence of antibodies against C. burnetii was high in South Limburg in the 1980s, even though no $\mathrm{Q}$ fever outbreaks were described in that period. Between 1987 and 2008, only three cases of acute Q fever were notified in South Limburg (1999, 2002, 2007). During this period, the 


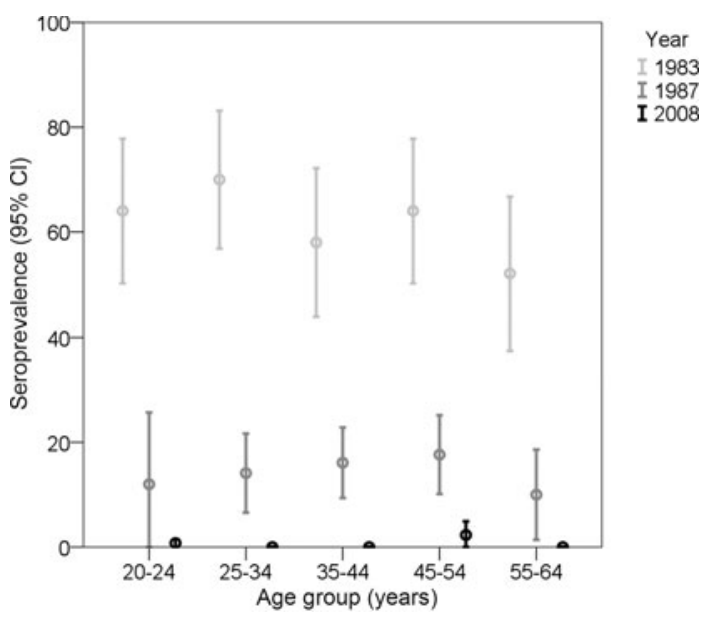

Fig. 2. Comparison of seroprevalence of $\mathrm{IgG}$ phase II-specific antibodies to Coxiella burnetii (Q fever) in Maastricht in 1983, 1987, and 2008.

seroprevalence declined in this geographical area. Since 2008 the number of notifications increased with a peak of 238 cases in 2009 (Table 2). We suggest that waning immunity in the population may have contributed to the size of the Q fever outbreak that occurred in South Limburg in 2009.

In interpreting these findings, several limitations have to be considered. First, by comparing results from historical seroprevalence surveys, we must account for different methodologies and study populations. Original sera from the published 1980s' seroprevalence studies were no longer available and the in-house IFA employed at the time could not be reproduced. Therefore, we tested historical samples from 1987 using a contemporary commercial IFA. We used a cut-off of 1:32, whereas a cut-off of 1:16 was used in the 1983 survey. Assuming that the performance of both IFAs was otherwise comparable, a lower cut-off with increased sensitivity and decreased specificity may have led to more false-positive results and an overestimation of seroprevalence in 1983. Moreover, the samples from 1983 and 1987 were collected in different ways. Whereas the samples from 1987 were (retrospectively) randomly selected, the 1983 specimens were selected such that each age group would include 50 samples. However, no significant differences were found in the age/gender distribution for both $1983(P=0.999)$ and $1987(P=0.856)$. The plasma samples we used for retrospective testing were stored for many years at a temperature of $-35^{\circ} \mathrm{C}$. The effect of this temperature on the survival of antibodies is unknown, but degradation of antibodies
Table 2. Number of notifications of acute $Q$ fever in the South Limburg region, 1988-2014

\begin{tabular}{llll}
\hline \hline Year & $\begin{array}{l}\text { No. of } \\
\text { notifications }\end{array}$ & Year & $\begin{array}{l}\text { No. of } \\
\text { notifications }\end{array}$ \\
\hline $1988^{*}$ & 0 & 2002 & 1 \\
1989 & 0 & 2003 & 0 \\
1990 & 0 & 2004 & 0 \\
1991 & 0 & 2005 & 0 \\
1992 & 0 & 2006 & 0 \\
1993 & 0 & 2007 & 1 \\
1994 & 0 & 2008 & 5 \\
1995 & 0 & 2009 & 238 \\
1996 & 0 & 2010 & 20 \\
1997 & 0 & 2011 & 2 \\
1998 & 0 & 2012 & 0 \\
1999 & 1 & 2013 & 1 \\
2000 & 0 & 2014 & 0 \\
2001 & 0 & & \\
\hline \hline
\end{tabular}

Data from RIVM-OSIRIS.

* Surveillance data for acute Q fever is only available from 1988 onwards.

could have led to underestimation of the true seroprevalence.

The low cut-off that was used in 1983 and the unknown test qualities of the in-house IFA, suggest that the 1983 seroprevalence of $60 \%$ might have been overestimated. However, the seroprevalence of $14.4 \%$ we found for 1987 is still markedly high. By contrast, during the Dutch $\mathrm{Q}$ fever epidemic, in high-incidence areas seroprevalence surveys in pregnant women in 2008, blood donors in 2009, and persons with risk factors for chronic $\mathrm{Q}$ fever found a mean seroprevalence of $9 \cdot 1 \%, 12 \cdot 2 \%$ and $10 \cdot 7 \%$, respectively $[8-10]$.

The seroprevalence estimate for 1987, based on the present analysis, is also significantly higher than the estimates based on samples from 2008 and 2010 from the same region. The samples from 2008 and 2010 were tested using a different IFA with a lower cut-off of 1:16, but this was according to the manufacturer's instructions. Further, the low seroprevalence estimates for 2008 and 2010 are comparable with the estimate in the study of Schimmer et al. [4], in which the IFA of Focus Diagnostics was used. In the Schimmer et al. study, the estimated seroprevalence increased from $1.5 \%$ to $2 \cdot 4 \%$ after correction for false-negative ELISA results. Therefore, such a correction of the 2008 and 2010 results might have led to a small increase in seroprevalence.

We have no clear explanation for the high C. burnetii seroprevalence in the 1980s, without much clinical $\mathrm{Q}$ fever disease at the time. One possibility is that the 
Table 3. The seroprevalence of antibodies against Coxiella burnetii in other European countries

\begin{tabular}{|c|c|c|c|c|}
\hline Year [reference] & Study population & $\begin{array}{l}\text { Testing assay } \\
\text { (cut-off titre) }\end{array}$ & $\begin{array}{l}\text { No. of } \\
\text { samples }\end{array}$ & $\begin{array}{l}\text { Positive samples } \\
n(\%)\end{array}$ \\
\hline \multicolumn{5}{|l|}{ Germany } \\
\hline $1977[18]$ & General population from the State of Hessen & CFT $(>10)$ & 1600 & $0(0 \cdot 0)$ \\
\hline $1983[19]$ & $\begin{array}{l}\text { Inhabitants of Seebron (State of } \\
\text { Baden-Württemberg) }\end{array}$ & $\mathrm{CFT}(\geqslant 5)$ & 715 & $136(19 \cdot 0)$ \\
\hline $1986[20]$ & Blood donors from the State of Hessen & ih-ELISA & 205 & $31(15 \cdot 1)$ \\
\hline $1987[21]$ & Blood donors from the State of Hessen & ih-ELISA & 207 & $32(15 \cdot 5)$ \\
\hline $2009^{*}$ & $\begin{array}{l}\text { General population from the State of } \\
\text { Baden-Württemberg }\end{array}$ & cELISA + cIFA & 1036 & $78(7 \cdot 5)$ \\
\hline \multicolumn{5}{|l|}{ France } \\
\hline 1986 [22] & Blood donors from southern France & ih-IFA $(\geqslant 32)$ & 325 & $17(5 \cdot 2)$ \\
\hline $1988[23]$ & Blood donors from Marseille & ih-IFA $(\geqslant 100)$ & 942 & $38(4 \cdot 0)$ \\
\hline $1996[24]$ & Pregnant women from southern France & ih-IFA $(\geqslant 100)$ & 12716 & $19(0 \cdot 2)$ \\
\hline $1996[25]$ & General population of Briançon & ih-IFA $(\geqslant 50)$ & 785 & $42(5 \cdot 4)$ \\
\hline $2002[26]$ & $\begin{array}{l}\text { Tested patients without risk factors in the } \\
\text { Chamonix Valley }\end{array}$ & ih-IFA $(\geqslant 100)$ & 578 & $31(5 \cdot 4)$ \\
\hline \multicolumn{5}{|c|}{ 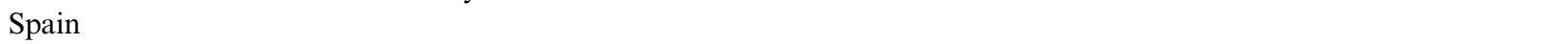 } \\
\hline 1983 [27] & Inhabitants of the Basque Country & CFT $(\geqslant 8)$ & 308 & $38(12 \cdot 3)$ \\
\hline $1985[27]$ & Inhabitants of the Basque Country & $\mathrm{CFT}(\geqslant 8)$ & 1180 & $199(16 \cdot 9)$ \\
\hline $1988[27]$ & Inhabitants of Barcelona & $\mathrm{CFT}(\geqslant 8)$ & 1016 & $91(9 \cdot 0)$ \\
\hline $1991[28]$ & Inhabitants of the Basque Country & $\operatorname{cIFA}(\geqslant 20)$ & 810 & $262(32 \cdot 3)$ \\
\hline $1993[29]$ & Inhabitants of Soria & $\operatorname{cIFA}(\geqslant 80)$ & 298 & $62(20 \cdot 8)$ \\
\hline $1994[27]$ & Inhabitants of the province of Léon & $\operatorname{cIFA}(\geqslant 80)$ & 406 & $165(40 \cdot 6)$ \\
\hline $1995[30]$ & Population of Eastern Cantabria & $\operatorname{cIFA}(\geqslant 16)$ & 595 & $289(48 \cdot 6)$ \\
\hline $2005[31]$ & Random patients from Barcelona & $\operatorname{cIFA}(\geqslant 40)$ & 216 & $33(15 \cdot 3)$ \\
\hline \multicolumn{5}{|c|}{ 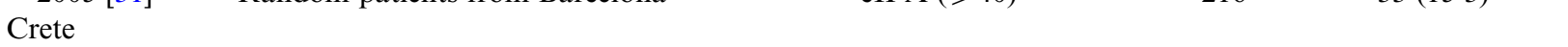 } \\
\hline 1985 [32] & Population of Anogia & $\operatorname{cIFA}(\geqslant 60)$ & 231 & $80(34 \cdot 6)$ \\
\hline $1985[32]$ & Population of Tymbaki & $\operatorname{cIFA}(\geqslant 60)$ & 188 & $11(5 \cdot 9)$ \\
\hline $1987[32]$ & Population of Anogia & $\operatorname{cIFA}(\geqslant 60)$ & 231 & $88(38 \cdot 1)$ \\
\hline $1987[32]$ & Population of Tymbaki & $\operatorname{cIFA}(\geqslant 60)$ & 188 & $26(13 \cdot 8)$ \\
\hline $1998[33]$ & Population of Anogia & $\operatorname{cIFA}(\geqslant 120)$ & 238 & $100(42 \cdot 0)$ \\
\hline $2006[34]$ & Healthy blood donors from Crete & $\operatorname{cIFA}(\geqslant 120)$ & 493 & $240(48 \cdot 7)$ \\
\hline \multirow{2}{*}{\multicolumn{5}{|c|}{$\begin{array}{l}\text { England and } \\
\text { Wales }\end{array}$}} \\
\hline & & & & \\
\hline $1966[35]$ & Patients not exposed to infection & $\mathrm{CFT}(\geqslant 20)$ & 7005 & $146(2 \cdot 1)$ \\
\hline $1987[36]$ & General population around Taunton & $\mathrm{CFT}(\geqslant 20)$ & 2773 & $52(1.9)$ \\
\hline $1989[37]$ & $\begin{array}{l}\text { Subgroup of emergency service workers } \\
\text { from Lancashire }\end{array}$ & ih-IFA $(\geqslant 32)$ & 395 & $43(10 \cdot 9)$ \\
\hline $1996[38]$ & Rural population from Wales & ih-IFA $(\geqslant 32)$ & 265 & $21(7 \cdot 9)$ \\
\hline 2008 [39] & Pregnant women around London & $\operatorname{cIFA}(\geqslant 50)$ & 438 & $20(4 \cdot 6)$ \\
\hline
\end{tabular}

ih-IFA, in-house developed indirect immunofluorescence assay; cIFA, commercial IFA; ih-ELISA, in-house developed enzyme-linked immunosorbent assay; cELISA, commercial ELISA; CFT, complement fixation test.

* Source: J. W. Brockman et al., unpublished observations.

high seroprevalence was due to exposure to a C. burnetii cattle genotype that might have been less virulent than the $C$. burnetii goat genotype that caused the large 2007-2010 epidemic in The Netherlands [11-14].

Using PCR testing, DNA from the cattle $C$. burnetii genotype can be found in retail milk and a possible hypothesis is that a daily dose of fragments of $C$. burnetii in retail milk could cause a seroconversion and immunize people rather than making them ill. In this context it would be interesting to study milk consumption patterns over time and changes that have taken place in milk pasteurization practices that in certain years may have been insufficient to kill $C$. burnetii. Another hypothesis is the spreading of the less virulent $C$. burnetii strain by dairy cattle farms. In The Netherlands, the number of dairy cattle declined while the dairy goat sector expanded rapidly after introduction of the European milk quota system in 1984 [15]. From 1984 to 2008, the number of cattle in 
South Limburg did decrease from 73366 to 36011 (data from Statistics Netherlands). This could have resulted in a lower exposure to the cattle $C$. burnetii genotype.

As shown in animal experiments, antibodies against C. burnetii protect against the development of $\mathrm{Q}$ fever after exposure to viable $C$. burnetii, probably due to enhanced macrophage activity [16]. Therefore, the decreasing seroprevalence of antibodies against $C$. burnetii in the regional population of South Limburg could have played a role in the regional outbreak that occurred in the region in 2009. The question remains whether the results for South Limburg are representative for the entire Dutch population. The high seroprevalence found in 1983 in other regions of The Netherlands [3] and the lack of differences in seroprevalence between regions in 2007 [4], suggest that seroprevalence decreased in the entire country. This could have made the entire Dutch population vulnerable for the almost nationwide outbreak of Q fever in the years 2007-2010.

Assuming a high risk for exposure and infection in the 1980s, followed by a period of low exposure, one would expect an age-cohort effect with higher seroprevalence in the older age groups, which indeed was demonstrated in a 2006/2007 survey [4]. The agecohort effect is further supported by the lack of an effect of age in the seroprevalence studies that were performed in the 1980s including our survey from 1987.

An interesting comparison can be made with the situation in the United States, where a national survey showed a seroprevalence of $3 \cdot 1 \%$, indicating that millions of people have been exposed to C. burnetii [17]. However, there are fewer than 100 notifications per year of human Q fever in the United States, suggesting gross underreporting and/or circulation of a less virulent (cattle) strain. Unfortunately, no historical seroprevalence results are known for the United States. For some European countries, historical data exist, as shown in Table 3. In some countries, a similar decrease in seroprevalence can be seen as for The Netherlands. However, the comparison of different seroprevalence surveys remains difficult, due to differences in the populations surveyed and developments in laboratory methods used since the 1980s. In contrast, in some high endemic areas, there seems to be an increase in seroprevalence.

\section{CONCLUSION}

C. burnetii seroprevalence in South Limburg declined from the 1980 s to 2008 . This might indicate waning population immunity that could have contributed to the 2009 Q fever outbreak is this region. A similar declining trend in the seroprevalence for other regions in The Netherlands, could possibly have contributed to the 2007-2010 epidemic. For a better understanding of the infection dynamics of $\mathrm{Q}$ fever and other infectious diseases, longitudinal studies over a longer period or repeated seroprevalence surveys with standardized serological methods will be necessary. We advocate a large multi-country study, with analyses performed by one reference centre.

\section{ACKNOWLEDGEMENTS}

This study was financed from the regular budget of the Centre for Infectious Disease Control of The Netherlands. We thank the technicians, Marsha Hesp and Carla Nijhuis from the Centre for Infectious Disease Control for testing the plasma samples. We also thank Robert Jan de Klein and colleagues for assisting in collection of plasma samples.

This research received no specific grant from any funding agency, commercial or not-for-profit sectors.

\section{DECLARATION OF INTEREST}

None.

\section{REFERENCES}

1. Maurin M, Raoult D. Q fever. Clinical Microbiology Reviews 1999; 12: 518-553.

2. Van der Hoek W, et al. Shifting priorities in the aftermath of a Q fever epidemic in 2007 to 2009 in the Netherlands: from acute to chronic infection. Eurosurveillance 2012; 17: 20059.

3. Richardus $\mathbf{J H}$, et al. Q fever in the Netherlands: a sero-epidemiological survey among human population groups from 1968 to 1983. Epidemiology and Infection 1987; 98: 211-219.

4. Schimmer B, et al. Low seroprevalence of Q fever in The Netherlands prior to a series of large outbreaks. Epidemiology and Infection 2012; 140: 27-35.

5. Blaauw GJ, et al. The application of an enzyme-linked immunosorbent assay or an immunofluorescent assay test leads to different estimates of seroprevalence of Coxiella burnetii in the population. Epidemiology and Infection 2012; 140: 36-41.

6. Hackert VH, et al. Q fever: single-point source outbreak with high attack rates and massive numbers of undetected infections across an entire region. Clinical Infectious Diseases 2012; 55: 1591-1599.

7. Verschuren WMM, et al. Prevalence of and changes in risk factors for cardiovascular diseases over the period 1987-1991. Final report of the Monitoring Project on 
Cardiovascular Disease Risk Factors 1987-1991. Bilthoven: RIVM, 1994, report no. 528901011.

8. Van der Hoek W, et al. Antibodies against Coxiella burnetii and pregnancy outcome during the 2007-2008 Q fever outbreaks in the Netherlands. BMC Infectious Diseases 2011; 11: 44.

9. Hogema BM, et al. Coxiella burnetii infection among blood donors during the 2009 Q-fever outbreak in The Netherlands. Transfusion 2012; 52: 144-150.

10. Kampschreur LM, et al. Screening for Coxiella burnetii seroprevalence in chronic Q fever high-risk groups reveals the magnitude of the Dutch Q fever outbreak. Epidemiology and Infection 2012: 1-5.

11. Tilburg JJ, et al. Genotypic diversity of Coxiella burnetii in the 2007-2010 Q fever outbreak episodes in the Netherlands. Journal of Clinical Microbiology 2012; 50: 1076-1078.

12. Roest HI, et al. Molecular epidemiology of Coxiella burnetii from ruminants in $\mathrm{Q}$ fever outbreak, the Netherlands. Emerging Infectious Diseases 2011; 17: 668-675.

13. Tilburg JJ, et al. Genotyping reveals the presence of a predominant genotype of Coxiella burnetii in consumer milk products. Journal of Clinical Microbiology 2012; 50: $2156-2158$.

14. Dijkstra F, et al. The 2007-2010 Q fever epidemic in the Netherlands: characteristics of notified acute $Q$ fever patients and the association with dairy goat farming. FEMS Immunology and Medical Microbiology 2012; 64: 3-12.

15. Roest HI, et al. The $\mathrm{Q}$ fever epidemic in the Netherlands: history, onset, response and reflection. Epidemiology and Infection 2011; 139: 1-12.

16. Humphres RC, Hinrichs DJ. Role of antibody in Coxiella burnetii infection. Infection and immunity 1981; 31: 641-645.

17. Anderson AD, et al. Seroprevalence of $\mathrm{Q}$ fever in the United States, 2003-2004. American Journal of Tropical Medicine and Hygiene 2009; 81: 691-694.

18. Schmatz HD, et al. Seroepidemiological investigations on the prevalence of rickettsial antibodies in man in the Federal Republic of Germany [in German]. Immunität und Infektion 1977; 5: 163-166.

19. Heinrich R, et al. Seroprevalence of Q fever in an endemic area of southern Germany [in German]. Deutsche medizinische Wochenschrift 1983; 108: 1318-1324.

20. Schmeer N, et al. Serodiagnosis of Q fever by enzymelinked immunosorbent assay (ELISA). Zentralblatt fur Bakteriologie, Mikrobiologie, und Hygiene. Series A 1987; 267: 57-63.

21. Frost JW, Hengst A. Seroepidemiological investigations on the presence of antibodies against $C$. burnetii in man in southern and middle Hessen [in German]. Tierärztliche Umschau 1993; 48: 148-154.

22. Raoult D, et al. Rickettsial antibody in southern France: antibodies to Rickettsia conorii and Coxiella burnetii among urban, suburban and semi-rural blood donors. Transactions of the Royal Society of Tropical Medicine and Hygiene 1987; 81: 80-81.
23. Tissot Dupont H, et al. Epidemiologic features and clinical presentation of acute $\mathrm{Q}$ fever in hospitalized patients: 323 French cases. American journal of medicine 1992; 93: 427-434.

24. Rey D, et al. Seroprevalence of antibodies to Coxiella burnetti among pregnant women in South Eastern France. European Journal of Obstetrics, Gynecology, and Reproductive Biology 2000; 93: 151-156.

25. Carrieri MP, et al. Investigation of a slaughterhouserelated outbreak of Q fever in the French Alps. European Journal of Clinical Microbiology \& Infectious Diseases 2002; 21: 17-21.

26. Tissot-Dupont H, et al. Role of sex, age, previous valve lesion, and pregnancy in the clinical expression and outcome of $\mathrm{Q}$ fever after a large outbreak. Clinical Infectious Diseases 2007; 44: 232-237.

27. Suarez-Estrada J, et al. Seroepidemiological survey of Q fever in Leon province, Spain. European Journal of Epidemiology 1996; 12: 245-250.

28. Sanzo JM, et al. Q fever: prevalence of antibodies to Coxiella burnetii in the Basque country. International Journal of Epidemiology 1993; 22: 1183-1188.

29. Saz JV, et al. Seroprevalence of Coxiella burnetii and Rickettsia conorii infection in the province of Soria [in Spanish]. Enfermedades infecciosas y microbiologia clinica 1993; 11: 469-473.

30. Pascual-Velasco F, et al. High seroprevalence of Coxiella burnetii infection in Eastern Cantabria (Spain). International Journal of Epidemiology 1998; 27: 142-145.

31. Cardenosa N, et al. Short report: seroprevalence of human infection by Coxiella burnetii in Barcelona (northeast of Spain). American Journal of Tropical Medicine and Hygiene 2006; 75: 33-35.

32. Antoniou M, et al. The seroprevalence of ten zoonoses in two villages of Crete, Greece. European Journal of Epidemiology 1995; 11: 415-423.

33. Antoniou M, et al. Fourteen-year seroepidemiological study of zoonoses in a Greek village. American Journal of Tropical Medicine and Hygiene 2002; 66: 80-85.

34. Vranakis I, et al. Serological survey of Q fever in Crete, southern Greece. Comparative Immunology, Microbiology and Infectious Diseases 2012; 35: 123-127.

35. Public Health Laboratory Service. The occurrence of Coxiella burnetii in North-Western England and North Wales. A report from five laboratories of the Public Health Laboratory Service. Journal of Hygiene 1969; 67: 125-133.

36. Jorm LR, Lightfoot NF, Morgan KL. An epidemiological study of an outbreak of Q fever in a secondary school. Epidemiology and Infection 1990; 104: 467-477.

37. Thomas DR, et al. The risk of acquiring Q fever on farms: a seroepidemiological study. Occupational and Environmental Medicine 1995; 52: 644-647.

38. Davies TR, et al. Prevalence of Q fever in a rural practice. Journal of Public Health Medicine 1997; 19: 324-327.

39. Baud D, et al. Seroprevalence of Coxiella burnetii and Brucella abortus among pregnant women. Clinical Microbiology and Infection 2009; 15: 499-501. 